\title{
Comparing the Outcomes of Ceftaroline Plus Vancomycin or Daptomycin Combination Therapy Versus Monotherapy in Adults with Complicated and Prolonged Methicillin-Resistant Staphylococcus Aureus Bacteremia Initially Treated with Supplemental Ceftaroline
}

\author{
Omar Ahmad (D) - Timothy N. Crawford · Thein Myint
}

Received: October 11, 2019 / Published online: November 28, 2019

(c) The Author(s) 2019

\section{ABSTRACT}

Introduction: Methicillin-resistant Staphylococcus aureus (MRSA) bacteremia confers considerable morbidity and mortality. Although vancomycin or daptomycin monotherapy is usually curative, prolonged bacteremia necessitating supplemental ceftaroline has occurred. The practice has led to the question of whether to continue with ceftaroline following bacteremia resolution.

Methods: Adult patients hospitalized with MRSA bacteremia at the University of Kentucky Medical Center between January 2015 and December 2017 were retrospectively reviewed. Study subjects required supplemental ceftaroline due to 4 or more days of bacteremia despite vancomycin or daptomycin. They additionally had accompanying native valve infective endocarditis, osteomyelitis, or brain abscess. Patients were divided into two cohorts. One

Enhanced Digital Features To view enhanced digital features for this article go to https://doi.org/10.6084/ m9.figshare.10303835.

O. Ahmad ( $\square) \cdot$ T. Myint

Division of Infectious Diseases, University of

Kentucky, Lexington, KY, USA

e-mail: oahmad3@gmail.com

T. N. Crawford

Department of Population and Public Health

Sciences, Wright State University, Dayton, OH, USA group continued with ceftaroline plus vancomycin or daptomycin following bacteremia resolution (combination therapy group). The other group received vancomycin or daptomycin alone (monotherapy group). All involved received 6-8 weeks of therapy. Patients' Pitt bacteremia score (PBS) and Charlson comorbidity index (CCI) values were calculated. Treatment outcomes of inpatient mortality, recurrence of bacteremia, 30-day readmission, acute kidney injury, and leukopenia were recorded and compared.

Results: A total of 30 patients comprised the study population. 15 patients were assigned to each cohort. The median PBS value of the combination therapy group was 2, compared with 1 among the monotherapy group. The median CCI score of both groups was 0 . No statistically significant difference in the aforementioned treatment outcomes was seen between the two groups.

Conclusion: In subjects with complicated and prolonged MRSA bacteremia requiring supplemental ceftaroline, clinical outcomes did not differ among patients prescribed vancomycin or daptomycin alone following bacteremia resolution versus patients who continued combination therapy.

Keywords: Bacteremia; Ceftaroline; Daptomycin; MRSA; Vancomycin 


\section{Key Summary Points}

\section{Why carry out this study?}

Supplemental ceftaroline is an established option in the treatment of prolonged MRSA bacteremia refractory to vancomycin or daptomycin therapy.

The question of whether to continue with ceftaroline plus vancomycin or daptomycin following bacteremia resolution, however, remains unanswered.

In hopes of uncovering findings supporting a particular regimen, treatment outcomes were compared in patients prescribed vancomycin or daptomycin alone following bacteremia resolution versus patients who continued combination therapy.

\section{What was learned from the study?}

No statistically significant difference in inpatient mortality, recurrence of bacteremia, 30-day readmission, acute kidney injury, or leukopenia was seen in patients prescribed vancomycin or daptomycin alone following bacteremia resolution versus patients who continued combination therapy.

Considering treatment outcomes did not differ in patients prescribed vancomycin or daptomycin alone following bacteremia resolution, our finding encourages confining ceftaroline use to active bacteremia.

\section{INTRODUCTION}

Methicillin-resistant Staphylococcus aureus (MRSA) bacteremia is a formidable disease associated with considerable morbidity and mortality [1,2]. Vancomycin or daptomycin has traditionally been the mainstay of therapy for invasive MRSA infection. However, treatment failures have happened in recent years, as defined by persistent bacteremia, bacteremia recurrence post-antimicrobial cessation, and/or death 30 days post-treatment [3]. Vancomycin's slow bactericidal activity, as well as the development of increased glycopeptide tolerance in MRSA isolates administered vancomycin, have been blamed for these occurrences $[4,5]$. The latter issue has also plagued daptomycin, with nonsusceptible isolates emerging during treatment being implicated in poor clinical outcomes [6].

In 2010, the Food and Drug Administration (FDA) approved the use of ceftaroline for the treatment of acute bacterial skin and skinstructure infections (ABSSSI) caused by MRSA. The antibiotic later received additional approval for the solitary treatment of ABSSSI-associated bacteremia [7]. Ceftaroline's value in treating MRSA bacteremia secondary to non-ABSSSI causes such as infective endocarditis and osteomyelitis $[8,9]$ led to its investigational use as a salvage measure for vancomycin- or daptomycin-refractory MRSA bacteremia $[9,10]$. Findings of bacteremia resolution plus in vitro evidence of antibiotic synergy $[11,12]$ motivated others to follow suit. Nowadays, it is common practice to add ceftaroline to patients with persistent bacteremia despite vancomycin or daptomycin.

The effectiveness of supplemental ceftaroline in treating refractory MRSA bacteremia is well established. However, the question of whether to continue with ceftaroline following bacteremia resolution remains unanswered. With this in mind, we studied adults with complicated and prolonged MRSA bacteremia prescribed ceftaroline plus vancomycin or daptomycin. We specifically compared treatment outcomes in those who continued with combination therapy following bacteremia resolution versus vancomycin or daptomycin alone in the hope of uncovering findings supporting a particular regimen.

\section{METHODS}

\section{Study Design and Subject Selection}

Adult patients (aged 21-99 years) hospitalized between January 1, 2015 and December 31, 
2017 at the University of Kentucky Medical Center with monomicrobial MRSA bacteremia were retrospectively identified. Subjects with bacteremia lasting 4 days or longer secondary to deep-seated infection i.e., native valve infective endocarditis (IE), osteomyelitis, and/or brain abscess underwent further analysis. Of these, only those who received supplemental ceftaroline following 4 or more days of bacteremia despite vancomycin or daptomycin were studied. Pregnant individuals, patients allergic to ceftaroline, and patients who were discharged against medical advice or had care withdrawn during their hospitalization, were excluded. Of note, none of the study subjects received antibiotics aside from vancomycin, daptomycin, or ceftaroline for more than $72 \mathrm{~h}$ following the detection of MRSA in blood culture specimens.

Included patients were separated into two treatment groups. One cohort comprised subjects who continued on ceftaroline plus either vancomycin or daptomycin following bacteremia resolution (combination therapy group). The other cohort (monotherapy group) comprised subjects who received vancomycin or daptomycin alone following bacteremia resolution, with ceftaroline being discontinued no later than $24 \mathrm{~h}$ after the first finalized negative blood culture set. All patients received 6-8 weeks of antimicrobial(s) upon bacteremia resolution due to their underlying infections.

Age, sex, comorbid conditions, length of MRSA bacteremia, and duration of antibiotics, as well as serum creatinine and white blood cell count results of subjects, were documented. Instances of death or bacteremia recurrence while hospitalized, along with instances of 30-day readmission, were recorded. Vital signs, need for intravenous vasopressor agents, need for mechanical ventilation, incidences of cardiac arrest, and patient mentation according to electronic medical record (EMR) documentation were noted the day of positive blood cultures.

The presented study received approval from the University of Kentucky Office of Research Integrity Institutional Review Board and conformed with the Helsinki Declaration of 1964 (as revised in 2013) concerning human and animal rights. On account of the investigation being carried out through retrospective review of medical records, ethics approval was not required due to no foreseeable impact on the rights and/or welfare of the subjects involved. Consent from study participants was additionally not obtained due to the study involving retrospective review of medical records.

\section{Antimicrobial Susceptibility Testing}

Two discrete methods of antimicrobial susceptibility testing identified MRSA isolates in the study. This was due to a procedural change midway through the trial period. Antimicrobial gradient diffusion testing, i.e., bioMérieux Etest $^{\mathrm{TM}}$, was employed between January 1, 2015 and March 31, 2016. During this time, S. aureus strains with a minimum inhibitory concentration (MIC) $\geq 4 \mu \mathrm{g} / \mathrm{mL}$ to oxacillin were classified as MRSA per Clinical and Laboratory Standards Institute (CLSI) criteria [13]. The BD Phoenix ${ }^{\mathrm{TM}}$ Automated Microbiology System was adopted thereafter. $S$. aureus strains identified as resistant to cefoxitin (defined by an MIC $\geq 8 \mu \mathrm{g} / \mathrm{mL}$ ) underwent corroborative disk diffusion testing. Those exhibiting an inhibition zone diameter $\leq 21 \mathrm{~mm}$ to cefoxitin were thereafter classified as MRSA per CLSI criteria [13].

Vancomycin and daptomycin susceptibility testing were also performed using Etest ${ }^{\mathrm{TM}}$ and Phoenix ${ }^{\mathrm{TM}}$ methods, in line with the time intervals previously mentioned. MRSA isolates with a MIC $\leq 2 \mu \mathrm{g} / \mathrm{mL}$ to vancomycin were deemed vancomycin-susceptible, and those with an $\mathrm{MIC} \leq 1 \mu \mathrm{g} / \mathrm{mL}$ to daptomycin were deemed daptomycin-susceptible. The designated breakpoints were shared by both Etest ${ }^{\mathrm{TM}}$ and Phoenix ${ }^{\mathrm{TM}}$. This aside, ceftaroline susceptibility testing was accomplished entirely by means of Etest ${ }^{\mathrm{TM}}$. MRSA isolates having an MIC $\leq 1 \mu \mathrm{g} / \mathrm{mL}$ to ceftaroline were labeled ceftaroline-susceptible.

No MRSA isolates in the study demonstrated daptomycin or ceftaroline resistance. Two isolates belonging to the combination therapy group developed borderline vancomycin susceptibility (MIC $=2 \mu \mathrm{g} / \mathrm{mL}$ ) in the midst of vancomycin therapy, i.e., MIC creep. The 
instances occurred while Etest ${ }^{\mathrm{TM}}$ was employed. However, no MRSA isolates were vancomycinintermediate or vancomycin-resistant according to CLSI definitions [13].

\section{Clinical Data Collection and Definitions}

Subjects with MRSA bacteremia were identified via institutional blood culture data. Episodes of native valve IE, osteomyelitis, or brain abscess were identified via chart review. Cases of native valve IE were validated provided they met Infectious Diseases Society of America (IDSA)-endorsed modified Duke criteria [14]. Instances of osteomyelitis or brain abscess were validated if diagnosed intraoperatively, or had compatible computed tomography/magnetic resonance imaging findings. Acute kidney injury (AKI) was diagnosed per standardized risk, injury, failure, loss of kidney function, and end-stage kidney disease criteria in patients without pre-existing end-stage renal disease. However, logistical constraints led to only serum creatinine determinants being utilized. In keeping with standardized laboratory parameters, leukopenia was characterized by a serum white blood cell count below $3.7 \mathrm{k} / \mu \mathrm{L}$. At least two consecutive eligible creatinine or white blood cell count values over a minimum span of $24 \mathrm{~h}$ were required for the respective diagnoses of AKI or leukopenia. This was to lessen the possibility of laboratory error.

Charlson comorbidity index (CCI) and Pitt bacteremia score (PBS) values were calculated using conventional scoring methods. Relevant medical conditions detailed in EMR documentation determined individual CCI values [15]. PBS values incorporated patient clinical status, medical needs, and documented vital signs within $24 \mathrm{~h}$ of bacteremia detection. The highest computed amount was ultimately selected for analysis [16].

\section{Antimicrobial Dosing}

Parenteral vancomycin was empirically dosed at $15-20 \mathrm{mg} / \mathrm{kg}$ every $8-12 \mathrm{~h}$. The specific regimen was dependent upon individual renal function. Dosing was thereafter adjusted to attain IDSArecommended trough levels of $15-20 \mu \mathrm{g} / \mathrm{mL}$
[17]. Daptomycin was dosed at $8-10 \mathrm{mg} / \mathrm{kg}$ once daily. This high-dose regimen was chosen over conventional $6 \mathrm{mg} / \mathrm{kg}$ daily dosing due to a reduced risk of antimicrobial resistance and the potential for accelerated bacterial clearance $[18,19]$. Subjects with a creatinine clearance $(\mathrm{CrCl})$ above $50 \mathrm{~mL} / \mathrm{min}$ were prescribed $600 \mathrm{mg}$ of intravenous ceftaroline every 8-12 h. Patients otherwise were dosed based on their calculated $\mathrm{CrCl}$ in accordance with FDA recommendations [20].

\section{Statistical Analysis}

Data were analyzed using SAS v.9.4 (Cary, NC, USA). $p$ values $<0.05$ were regarded as statistically significant. Descriptive statistics were calculated with means, standard deviations, medians, and interquartile ranges for all continuous variables; frequencies and percentages for all categorical variables. To examine bivariate associations with drug type, $\chi^{2}$ tests and Fisher's Exact tests were conducted for categorical variables, and $t$ tests and Mann-Whitney $U$ tests were conducted for continuous variables.

Inverse probability of treatment weighting (IPTW) was utilized to reduce potential bias and confounding by indication. Patient propensity scores were derived via logistic regression and incorporated age, CCI, and PBS values as covariates.

\section{RESULTS}

\section{Patient Selection}

During the study period, we identified 263 patients with MRSA bacteremia lasting 4 days or longer. All were prescribed either vancomycin or daptomycin. One hundred twenty-three of these patients were later prescribed supplemental ceftaroline. Seven patients, however, were removed from study consideration due to ceftaroline being started within 3 days of bacteremia onset.

Twenty-eight of the remaining patients were excluded due to the absence of underlying native valve IE, osteomyelitis, or brain abscess. 
Three patients were additionally excluded due to care being withdrawn during their hospital stay. Finally, 5 patients were excluded due to leaving against medical advice. In the end, 80 subjects constituted our preliminary investigational population.

Fifty patients were later removed from the study. Specifically, 8 patients were excluded due to dying prior to bacteremia resolution. Twentyfive patients were additionally excluded due to being interhospital transfers with pre-existing MRSA bacteremia-thereby preventing requisite PBS value calculation. Finally, 17 patients were excluded due to being prescribed discordant antimicrobial regimens following bacteremia resolution. This resulted in a final study population of 30 subjects (Fig. 1).

\section{Monotherapy Group Patient Characteristics and Outcomes}

Fifteen patients ( 8 males, 7 females) comprised the monotherapy cohort. Ages ranged from 25 to 70 years old, with an average age of 41 . Thirteen subjects had native valve IE (87\%), 1 subject had osteomyelitis (7\%), and 1 subject was diagnosed with both conditions (7\%). Two of the 12 patients (17\%) with native valve IE warranting early valve surgery [14] underwent valve replacement while bacteremic. Ceftaroline was begun within 4 to 11 days of refractory bacteremia, with a median value of 6 days. Bacteremia duration varied between 4 and 11 days, with a median value of 7 days. PBS values ranged from 0 to 6 , corresponding to a median value of 1 . CCI scores were between 0 and 5 , with a median score of 0 (Table 1 ).

Vancomycin was initially prescribed to all subjects. Five patients, however, were later changed to daptomycin. This was due to AKI in 3 patients. The other 2 patients were changed to daptomycin on account of provider preference. In terms of treatment duration, 2 patients received 7 weeks of therapy and 12 subjects received 6 weeks of therapy. This amounted to a median value of 6 weeks.

There were no incidences of leukopenia in the monotherapy group. Six patients were diagnosed with AKI. None, however, required hemodialysis at the time of discharge. Two instances of 30-day readmission occurred, though they were unrelated to patients' previous hospitalizations. One was a result of polymerase chain reaction-confirmed human metapneumovirus pneumonia. The other was to prevent an interruption in outpatient parenteral antimicrobial therapy. One subject encountered a brief 2-day period of bacteremia recurrence prior to ceftaroline discontinuation. It occurred as vancomycin was replaced with daptomycin due to vancomycin-induced AKI. This led to Ceftaroline being administered alone for approximately $12 \mathrm{~h}$. Considering the circumstances, the bacteremia recurrence seemingly was provoked by treatment interruption as opposed to deficient therapy.

One patient in the monotherapy group expired. A 33-year-old female with native valve IE, she had an abrupt decline status-post bioprosthetic mitral valve replacement ( 5 days after bacteremia resolution). She was diagnosed with obstructive shock secondary to a post-operative pericardial thrombus. Despite undergoing emergent mediastinal exploration with successful evacuation of the clot, she ultimately arrested. No autopsy was performed following her death.

\section{Combination Therapy Group Patient Characteristics and Outcomes}

Fifteen patients (10 males, 5 females) comprised the combination therapy cohort. Ages ranged from 24 to 73 years old, with an average age of 46. Seven subjects had osteomyelitis $(47 \%), 5$ subjects had native valve IE (33\%), 2 subjects were diagnosed with both conditions (13\%), and 1 person had a cerebral abscess (7\%). One of the 7 patients (14\%) with native valve IE warranting early valve surgery [14] underwent valve replacement while bacteremic. Ceftaroline was begun within 4 to 10 days of refractory bacteremia, with a median value of 6 days. Bacteremia duration varied between 5 and 14 days, with a median value of 8 days. PBS values ranged from 0 to 6 , corresponding to a median value of 2 . CCI scores were between from 0 and 3 , with a median score of 0 (Table 1 ). 


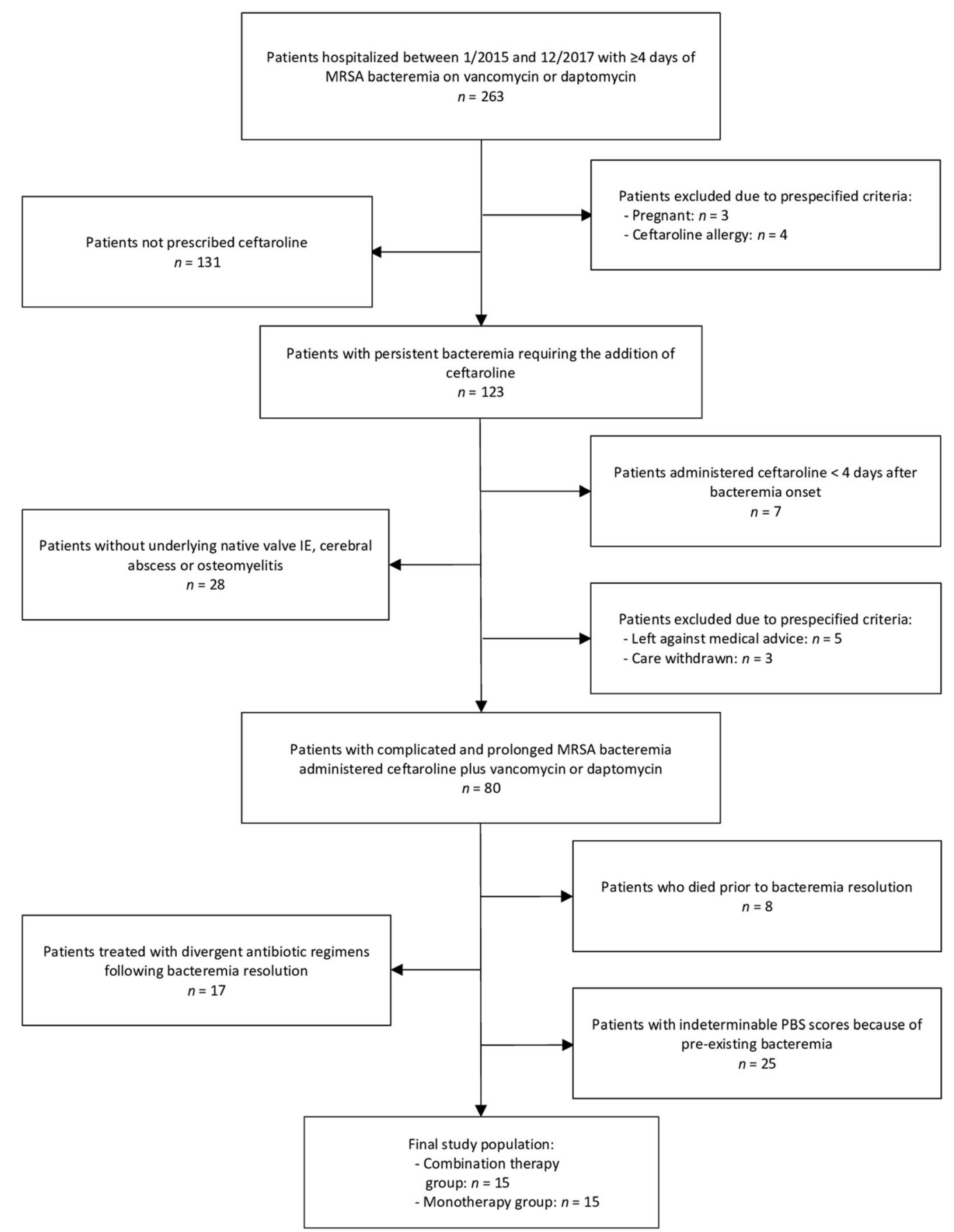

Fig. 1 Enrollment flow chart and inclusion/exclusion criteria

Vancomycin was initially prescribed to all subjects. Twelve patients, however, were later changed to daptomycin. This was due to the emergence of borderline vancomycin-susceptible MRSA strains in 2 patients, AKI in one person, an allergic reaction in one person, drug- induced neutropenia in one person, and an inability to attain a consistent vancomycin trough of $15-20 \mathrm{ug} / \mathrm{mL}$ in one person. The other 6 patients were changed to daptomycin on account of provider preference. In terms of treatment duration, 3 patients received 8 weeks 
Table 1 Patient characteristics and study outcomes

\begin{tabular}{|c|c|c|c|}
\hline Variable & $\begin{array}{l}\text { Monotherapy group } \\
(n=15)\end{array}$ & $\begin{array}{l}\text { Combination therapy group } \\
(n=15)\end{array}$ & $p$ value \\
\hline Age (years), mean (SD) & $41(13.6)$ & $46(15.9)$ & 0.42 \\
\hline Sex, $n(\%)$ & & & 0.71 \\
\hline Female & $7(47)$ & $5(33)$ & \\
\hline Male & $8(53)$ & $10(67)$ & \\
\hline \multicolumn{4}{|l|}{ Accompanying infections, $n(\%)$} \\
\hline Osteomyelitis & $1(7)$ & $7(47)$ & 0.04 \\
\hline Native valve IE & $13(87)$ & $5(33)$ & 0.01 \\
\hline Osteomyelitis and native valve IE & $1(7)$ & $2(13)$ & 0.99 \\
\hline Cerebral abscess & $0(0)$ & $1(7)$ & 0.99 \\
\hline $\begin{array}{l}\text { Surgical candidates who underwent valve } \\
\text { replacement, } n(\%)\end{array}$ & $2(17)$ & $1(14)$ & 0.99 \\
\hline PBS score, median (IQR) & $1(4.0)$ & $2(4.0)$ & 0.61 \\
\hline CCI score, median (IQR) & $0(1.0)$ & $0(2.0)$ & 0.85 \\
\hline $\begin{array}{l}\text { Bacteremia duration preceding ceftaroline (days), } \\
\text { median (IQR) }\end{array}$ & $6(2.0)$ & $6(3.0)$ & 0.85 \\
\hline Total bacteremia duration (days), median (IQR) & $7(3.0)$ & $8(4.0)$ & 0.22 \\
\hline Treatment duration (weeks), median (IQR) & $6(0)$ & $6(1.5)$ & 0.39 \\
\hline MRSA strains with $\mathrm{MIC}=2 \mu \mathrm{g} / \mathrm{mL}$ & 2 & 0 & 0.48 \\
\hline \multicolumn{4}{|l|}{ Clinical outcomes, $n(\%)$} \\
\hline AKI & $6(40)$ & $7(47)$ & 0.36 \\
\hline Leukopenia & $0(0)$ & $1(7)$ & 0.35 \\
\hline Bacteremia recurrence & $1(7)$ & $0(0)$ & 0.27 \\
\hline 30-Day readmission & $2(13)$ & $0(0)$ & 0.14 \\
\hline Death & $1(7)$ & $3(20)$ & 0.24 \\
\hline
\end{tabular}

$A K I$ acute kidney injury, CCI Charlson comorbidity index, IE infective endocarditis, IQR interquartile range, $M I C$ minimum inhibitory concentration, MRSA methicillin-resistant Staphylococcus aureus, PBS Pitt bacteremia score, SD standard deviation

of therapy and 9 patients received 6 weeks of therapy. This amounted to a median value of 6 weeks.

There was an instance of leukopenia in one person belonging to the combination therapy group. A mild aberration, it led to no particular intervention or detriment. Seven patients were diagnosed with AKI-2 of whom required continued hemodialysis upon hospital discharge. No instances of 30-day readmission or bacteremia recurrence occurred.

Three patients in the combination therapy group expired. The first subject was a 40 -yearold male with native valve IE who died 6 days following bacteremia resolution. Suffering from septic central nervous system emboli with 
hemorrhagic manifestations (first detected 3 days after admission), he expired due to subsequent uncal herniation. The second subject was a 36-year-old female with native valve IE who died 26 days following bacteremia resolution. She progressed well clinically up until sudden hypoxemia led to the diagnosis of acute pulmonary embolism with right heart strain. Despite anticoagulation and aggressive life support measures, she expired within $48 \mathrm{~h}$. The third subject was a 69-year-old male with both native valve IE and osteomyelitis who died 23 days following bacteremia resolution. A few days after his unrivaled 14-day duration of bacteremia ended, acute hypoxemia led to the discovery of aortic valve perforation via echocardiography. He was deemed to not be a surgical candidate by cardiothoracic surgery on the account of clinical instability. He eventually expired due to progressive cardiac decompensation refractory to medical therapy. Of note, none of the above patients underwent autopsy.

\section{Group Outcome Comparisons}

No statistically significant difference in patient outcomes was seen after comparing the two treatment groups following IPTW standardization (Table 1). Bacteremia recurrence occurred in 1 person in the monotherapy group (7\%) versus 0 in the combination therapy group $(p=0.27)$. Two cases of 30-day readmission occurred in the monotherapy group (13\%) versus 0 in the combination therapy group $(p=0.14)$. While 3 patients died in the combination therapy group (20\%) versus only 1 in the monotherapy group (7\%), the difference was not statistically significant $(p=0.24)$. A total of 6 instances of AKI occurred in the monotherapy group (40\%) versus 7 in the combination therapy group (47\%) $(p=0.36)$. Finally, 1 episode of leukopenia occurred in the monotherapy group (7\%) versus 0 in the combination therapy group $(p=0.35)$.

\section{DISCUSSION}

To the best of our knowledge, this is the first published study investigating whether to continue with ceftaroline following bacteremia resolution in patients with prolonged MRSA bacteremia requiring combination therapy. As detailed previously, no statistically significant difference in treatment outcomes was seen between the combination therapy and monotherapy groups. The result complements an in vitro study reported by Barber [21] which compared MRSA isolates treated with daptomycin plus ceftaroline for 8 days to MRSA isolates treated with daptomycin plus ceftaroline for 4 days followed by a 4-day course of daptomycin alone. Pharmacokinetic/pharmacodynamic analysis found no significant difference in growth inhibition between the two regimens.

Considering treatment outcomes did not differ in patients prescribed vancomycin or daptomycin alone following bacteremia resolution, our finding encourages confining ceftaroline use to active bacteremia. It relatedly affords beneficial medical and financial repercussions. Adverse effects of ceftaroline ranging from mild intolerance, e.g., headache, nausea, diarrhea, etc. to worrisome Clostridioides difficile infection have been reported [22].Given this, requisite employment seems prudent from a patient safety standpoint. The perceived threat of future antimicrobial resistance further compels economical usage. Discontinuing ceftaroline following bacteremia resolution also potentially eliminates several weeks of ceftaroline therapy. In so doing, it allows for substantial cost savings individually as well as institutionally.

No universal length-related definition of prolonged MRSA bacteremia exists. We chose to investigate patients administered ceftaroline after at least 4 days of MRSA bacteremia given said duration has been associated with an increased risk of mortality [23]. This aside, there were concerns that patient degree of comorbidities and/or illness severity influenced medical providers' decision to continue with ceftaroline following bacteremia resolution. Representative CCI and PBS values along with subject ages were consequently used in propensity score formulation. IPTW standardization of the trial population was thereafter implemented to diminish the likelihood of indication bias compromising our findings.

Regarding study deaths, the occurrences seemingly were not due to substandard 
antibiotic therapy. The one monotherapy cohort patient likely instead expired from noninfectious complications of valve surgery. As for deaths in the combination therapy cohort, the 40 -year-old male patient with native valve IE likely expired from medically unpreventable/ untreatable disease sequelae given his pre-existing cerebral emboli. The 36-year-old female patient with native valve IE likely expired from a known consequence of critical illness-given her fatal acute pulmonary embolism. Finally, the 69-year-old male with both native valve IE and osteomyelitis likely expired from an unfortunate combination of disease severity plus inadequate source control given the protracted duration of his preceding bacteremia. However, evidence in support of these conclusions is unable to be provided due to absent autopsy results.

Our study is not without a few limitations. Aside from the innate flaws associated with any retrospective investigation, the study population was admittedly modest. This was partly due to infrequent episodes of prolonged MRSA bacteremia necessitating ceftaroline at our institution. Two stipulations additionally pared the study census. A significant number of patients were excluded from study consideration due to absent accompanying osteomyelitis, native valve IE, or brain abscess. These requirements, though, were essential in ensuring subjects appropriately received several weeks of antimicrobial therapy. It was feared that shorter antibiotic courses could possibly obscure outcome differences between the treatment groups. Secondly, many patients were transferred from neighboring medical centers with known MRSA bacteremia. Limited accompanying medical records/patient data precluded PBS score calculation. The subsequent inability to eliminate severity of illness as a confounding factor warranted their removal.

Another study limitation pertained to patient deaths. As mentioned previously, three subjects were removed from the study population due to care being withdrawn during their hospital stay. This was necessary in maintaining study integrity. That being said, these patients all had a minimal chance for recovery. Study deaths may thus have been lessened by their removal.

Although not a limitation per se, daptomycin replaced vancomycin in several patients once ceftaroline was begun. The change in therapy was largely due to AKI or provider preference. All involved MRSA isolates retained vancomycin susceptibility at the time. In this respect, the antibiotic change likely was inconsequential as both agents equally treat vancomycin-susceptible MRSA bacteremia [4]. As for the two combination therapy group patients prescribed daptomycin due to emergent borderline vancomycin-susceptible MRSA strains, fears of vancomycin ineffectiveness led to the substitution. Whether MIC creep absolutely demands vancomycin discontinuation in patients with MRSA bacteremia is unclear [26]. Regardless, the change in therapy likely did not affect assessed patient outcomes. The fact that it preceded bacteremia resolution and daptomycin was continued (along with ceftaroline) for $6-8$ weeks as planned supports this conclusion.

The PBS model was chosen for risk stratification purposes as opposed to alternative scoring systems due to it being developed expressly for bacteremic subjects. The decision was further supported by the studies of Gasch [24] and Kim [25], which found PBS values greater than 3 to be associated with early mortality in patients with MRSA bacteremia. This gave context to our patients' PBS values given that no universal score interpretation exists. The median PBS value of the combination therapy cohort was 2 and the median PBS value of the monotherapy cohort was 1. As such, both treatment groups lacked severely ill patients. This raises the question of whether our findings apply to sicker individuals. Also, the study population largely consisted of middle-aged subjects with no past medical history aside from intravenous drug abuse. The absence of subjects with multiple chronic medical problems led to a median CCI score of 0 for both cohorts. This raises the question of whether our findings apply to individuals with considerable comorbid conditions. 


\section{CONCLUSION}

In summary, discontinuing ceftaroline following bacteremia resolution in patients with vancomycin- or daptomycin-refractory complicated and prolonged MRSA bacteremia resulted in no statistically significant difference in treatment outcomes versus patients who continued combination therapy. This finding suggests restricting supplemental ceftaroline to bacteremic patients. However, given the retrospective nature of our study and overall patient characteristics, future prospective trials involving more ill subjects with several chronic diseases would be worthwhile.

\section{ACKNOWLEDGEMENTS}

We thank the patients who participated in the study.

Funding. No funding or sponsorship was received either for the study or article publication. The Rapid Service Fee was funded by the authors.

Editorial Assistance. We would like to thank Susan Shepherd, MS, for her assistance in drafting the antimicrobial susceptibility testing section of the manuscript.

Authorship. All named authors meet the International Committee of Medical Journal Editors (ICMJE) criteria for authorship of this article, take responsibility for the integrity of the work as a whole, and have given their approval for this version to be published.

Disclosures. Omar Ahmad, Timothy N. Crawford and Thein Myint declare that they have no conflict of interest.

Compliance with Ethics Guidelines. The presented study received approval from The University of Kentucky Office of Research Integrity Institutional Review Board and conformed with the Helsinki Declaration of 1964 (as revised in 2013) concerning human and animal rights. On account of the investigation being carried out through retrospective review of medical records, ethics approval was not required due to no foreseeable impact on the rights and/or welfare of subjects involved. Consent from study participants was additionally not obtained due to the study solely involving retrospective review of medical records.

Open Access. This article is distributed under the terms of the Creative Commons Attribution-NonCommercial 4.0 International License (http://creativecommons.org/licenses/ by-nc/4.0/), which permits any noncommercial use, distribution, and reproduction in any medium, provided you give appropriate credit to the original author(s) and the source, provide a link to the Creative Commons license, and indicate if changes were made.

\section{REFERENCES}

1. Mylotte JM, McDermott C, Spooner JA. Prospective study of 114 consecutive episodes of Staphylococcus aureus bacteremia. Rev Infect Dis. 1987;9:891.

2. Shurland S, Zhan M, Bradham DD, Roghmann MC. Comparison of mortality risk associated with bacteremia due to methicillin-resistant and methicillin-susceptible Staphylococcus aureus. Infect Control Hosp Epidemiol. 2007;28:273.

3. Lodise TP, Graves J, Evans A, Graffunder E, Helmecke M, Lomaestro BM, Stellrecht K. Relationship between vancomycin MIC and failure among patients with methicillin-resistant Staphylococcus aureus bacteremia treated with vancomycin. Antimicrob Agents Chemother. 2008;52:3315.

4. Kollef MH. Limitations of vancomycin in the management of resistant staphylococcal infections. Clin Infect Dis. 2007;45:191-5.

5. Sakoulas G, Moellering RC. Increasing antibiotic resistance among methicillin-resistant Staphylococcus aureus strains. Clin Infect Dis. 2008;46:360-7.

6. Boucher HW, Sakoulas G. Perspectives on daptomycin resistance, with emphasis on resistance in Staphylococcus aureus. Clin Infect Dis. 2007;45: 601-8. 
7. Corey GR, Wilcox M, Talbot GH, Friedland HD, Baculik T, Witherell GW, Critchley I, Das AF, Thye D. Integrated analysis of CANVAS 1 and 2: phase 3, multicenter, randomized, double-blind studies to evaluate the safety and efficacy of ceftaroline versus vancomycin plus aztreonam in complicated skin and skin-structure infection. Clin Infect Dis. 2010;51:641-50.

8. White BP, Barber KE, Stover KR. Ceftaroline for the treatment of methicillin-resistant Staphylococcus aureus bacteremia. Am J Health Syst Pharm. 2017;74:201-8.

9. Cosimi RA, Beik N, Kubiak DW, Johnson JA. Ceftaroline for severe methicillin-resistant Staphylococcus aureus infections: a systematic review. Open Forum Infect Dis. 2017;4:ofx084.

10. Gritsenko D, Fedorenko M, Ruhe JJ, Altshuler J. Combination therapy with vancomycin and ceftaroline for refractory methicillin-resistant Staphylococcus aureus bacteremia: a case series. Clin Ther. 2017;39:212-8.

11. Barber KE, Rybak MJ, Sakoulas GJ. Vancomycin plus ceftaroline shows potent in vitro synergy and was successfully utilized to clear persistent daptomycinnon-susceptible MRSA bacteremia. J Antimicrob Chemother. 2015;70:311-3.

12. Shafiq I, Bulman ZP, Spitznogle SL, Osorio JE, Reilly IS, Lesse AJ, Parameswaran GI, Mergenhagen KA, Tsuji BT. A combination of ceftaroline and daptomycin has synergistic and bactericidal activity in vitro against daptomycin nonsusceptible methicillin-resistant Staphylococcus aureus (MRSA). Infect Dis (Lond). 2017;49:410-6.

13. Clinical and Laboratory Standards Institute/NCCLS. Performance Standards for Antimicrobial Susceptibility Testing. Sixteenth informational supplement M100-S16. Wayne: CLSI; 2006.

14. Baddour LM, Wilson WR, Bayer AS, Fowler VG, Tleyjeh IM, Rybak MJ, Barsic B, Lockhart PB, Gewitz $\mathrm{MH}$, Levison ME, Bolger AF, Steckelberg JM, Baltimore RS, Fink AM, O'Gara P, Taubert KA. Infective endocarditis in adults: diagnosis, antimicrobial therapy, and management of complications: a scientific statement for healthcare professionals from the american heart association. Circulation. $2015 ; 132: 1435$.

15. Charlson ME, Pompei P, Ales KL, MacKenzie CR. A new method of classifying prognostic comorbidity in longitudinal studies: development and validation. J Chronic Dis. 1987;40:373-83.

16. Chow JW, Fine MJ, Shlaes DM, Quinn JP, Hooper DC, Johnson MP, Ramphal R, Wagener MM, Miyashiro DK, Yu VL. Enterobacter bacteremia: clinical features and emergence of antibiotic resistance during therapy. Ann Intern Med. 1991;115: 585-90.

17. Liu C, Bayer A, Cosgrove SE, Daum RS, Fridkin SK, Gorwitz RJ, Kaplan SL, Karchmer AW, Levine DP, Murray BE, Rybak M, Talan DA, Chambers HF. Clinical practice guidelines by the Infectious Diseases Society of America for the treatment of methicillin-resistant Staphylococcus aureus infections in adults and children. Clin Infect Dis. 2011;52:e18-55.

18. Wu G, Abraham T, Rapp J, Vastey F, Saad N, Balmir E. Daptomycin: evaluation of a high-dose treatment strategy. Int J Antimicrob Agents. 2011;38:192-6.

19. Bassetti M, Nicco E, Ginocchio F, Ansaldi F, de Florentiis D, Viscoli C. High-dose daptomycin in documented Staphylococcus aureus infections. Int J Antimicrob Agents. 2010;36:459-61.

20. Teflaro [package insert]. Saint Louis: Forest Pharmaceuticals; 2016.

21. Barber KE, Werth BJ, Rybak MJ. The combination of ceftaroline plus daptomycin allows for therapeutic de-escalation and daptomycin sparing against MRSA. J Antimicrob Chemother. 2015;70:505-9.

22. Duplessis C, Crum-Cianflone NF. Ceftaroline: a new cephalosporin with activity against methicillin-resistant Staphylococcus aureus (MRSA). Clin Med Rev Ther. 2011;3:a2466.

23. van Hal SJ, Jensen SO, Vaska VL, Espedido BA, Paterson DL, Gosbell IB. Predictors of mortality in Staphylococcus aureus bacteremia. Clin Microbiol Rev. 2012;25:362-86.

24. Gasch O, Camoez M, Dominguez MA, Padilla B, Pintado V, Almirante B, Lepe JA, Lagarde M, Ruiz de Gopegui E, Martínez JA, Montejo M, Torre-Cisneros J, Arnáiz A, Goenaga MA, Benito N, Rodríguez-Baño J, Pujol M. Predictive factors for early mortality among patients with methicillin-resistant Staphylococcus aureus bacteremia. J Antimicrob Chemother. 2013;68:1423-30.

25. Kim T, Chong YP, Park KH, Bang KM, Park SJ, Kim $\mathrm{SH}$, Jeong JY, Lee SO, Choi SH, Woo JH, Kim YS. Clinical and microbiological factors associated with early patient mortality from methicillin-resistant Staphylococcus aureus bacteremia. Korean J Intern Med. 2018;34:184-94.

26. Kalil AC, Van Schooneveld TC, Fey PD, Rupp ME. Association between vancomycin minimum inhibitory concentration and mortality among patients with Staphylococcus aureus bloodstream infections: a systematic review and meta-analysis. JAMA. 2014;312:1552-64. 